\title{
Egomotion Estimation as an Appearance-Based Classification Problem
}

\author{
Pedro Sánchez ${ }^{1}$, Cornelio Yáñez ${ }^{2}$, Jonathan Pecero³ , and Apolinar Ramírez ${ }^{1}$ \\ ${ }^{1}$ Instituto Tecnológico de Ciudad Madero, Tamaulipas, México \\ ${ }^{2}$ Instituto Politécnico Nacional, Centro de Investigación en Computación, D.F. México \\ ${ }^{3}$ Instituto Nacional Politécnico de Grenoble, France \\ sanchezorellana_pedro@yahoo.com.mx
}

\begin{abstract}
In this paper a probabilistic approach is considered to develop a methodology to solve the problem of estimation of the position of the observer. The base of this methodology is the appearance vision with which an environment map is constructed using Kernel PCA. For the experiments an image set is acquired in unknown locations in the same environment. The performance of Kernel PCA technique was tested according to the optimum dimension of the environment model and the quantity of images correctly classified using a Bayesian algorithm. To validate the results obtained with Kernel PCA the same experiments were performed with PCA and APEX techniques, then the results were compared showing that Kernel PCA has better performance than PCA and APEX.
\end{abstract}

Keywords: Egomotion estimation, probabilistic approach, Kernel PCA.

\section{Introduction}

One of the key tasks inside the systems of visual navigation is the estimation of the position of the observer, which allows an autonomous mobile robot to have constant feedback of its position regarding to a reference point in the environment. By means of this information a mobile robot can make more complex tasks like target pursuit or optimum route planning.

The estimate of the position has been approached using geometric focuses that allow to estimate the observer position calculating the rotation and translation speeds [1], [2], [3], [4], [5], [6], [7], nevertheless this approach requires the consideration of restrictions in the environment conditions, for example, illumination conditions must be uniform. In this work we use a probabilistic focus; the base of this focus is the use of appearance based models, where the appearances of the environment depends on the positions - the location $\mathrm{T}:(\mathrm{x}, \mathrm{y})$ and the orientation $\Omega-$, from which the observer captures images. This finite set of images is used to create an environment model, against which the current appearance of the environment (a new captured image) is compared in order to estimate the current position of the observer.

This approach has been applied to solve different problems, for example, object recognition [8], tracking of human figures [9] and face recognition [10]. However, the main complexity in this focus resides in the quantity of images (and the dimension of 
these) employed to create the environment model. This problem has been overcome by means of the use of techniques of compression of images like PCA (Principal Components Analysis) [11].

The contribution of this paper is the use of a variant of classic PCA known as Kernel PCA (Section 3 no. 2), which recently has been used to solve classification tasks, for example, face recognition [12], showing a better performance than classical compression techniques (PCA). In addition this technique is combined with a Bayesian algorithm and an Active Vision strategy (Section 3 no. 4), which allowed us to improve the obtained results.

\section{Formal Definition of the Egomotion Estimation from a Probabilistic Approach}

A sequence of images of an environment is obtained from $T=\left\{T_{1}, \ldots, T_{N}\right\}$ different locations. In each one of them a set of images is captured $\Omega=\left\{\Omega_{1}, \ldots, \Omega_{p}\right\}$, where $\Omega$ is a set of the possible orientations from a $T$-esim location, each orientation is separated $X$ degrees of the subsequent orientation, see Section 5.1, creating a database indexed by the $N$ locations. This database is used to create an environment model by means of the technique of principal components analysis "Kernel PCA". The probability that a new image $I_{A}$ is obtained in the same environment (environment model) in a location $T_{i}$ and with certain orientation $\Omega_{j}$ is given by eq. 1 [13].

$$
p\left(\Omega_{j}: T_{i} \mid I_{A}\right)
$$

Where $\Omega_{j}$ is the observer orientation in the environment $T_{i}$ is the location of the observer $(\mathrm{x}, \mathrm{y})$ in the environment and $I$ is an image captured by the observer in the current position in the environment.

Using the equation (1) the problem of estimating the position of the observer can be solved as an image classification problem; this means, now the objective is to classify a new image (obtained by the observer in an unknown position) into a set of known images.

Considering the a priori information (environment model) the current observer position $\left(\Omega_{j}: T_{i}\right)$ can be recovered, rewriting the equation (1) by means the Bayes theorem $p\left(I_{A} \mid \Omega_{j}: T_{i}\right)$ as it is shown in the (Section 3 no. 4).

\section{Solution Methodology}

The solution methodology consists of four steps (Fig. 1).

1. - Capture of images: The locations $(\mathrm{T})$ and the orientations $(\Omega)$ are defined for the capture of the gray tone images. These images are indexed for location so a location contains all the possible appearances of the environment from that specific point; that means, the appearance of the environment from a location $T_{i}$ is captured 
rotating the camera $X$ degrees and captures an image in each turn until completing the $360^{\circ}$. The obtained images are of dimension $60 \times 60$, each of those are vectorized, the resulting images are m-dimensional $(m=60 \times 60), x_{1}, \ldots, x_{n} \in \mathbb{R}^{m}$.

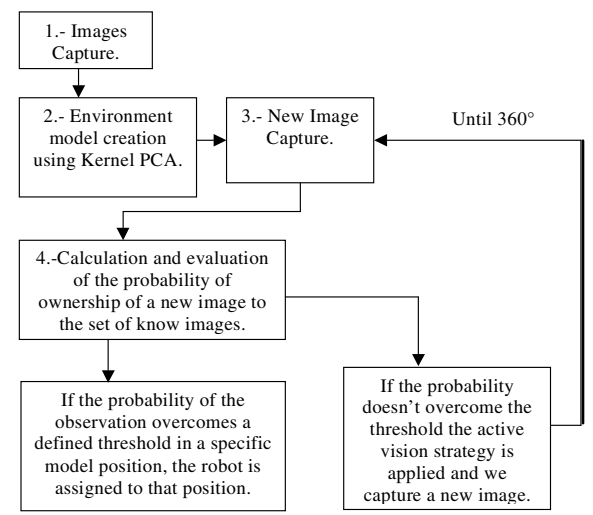

Fig. 1. Solution methodology

2. - The model of the environment: In order to deal with the amount of images and the dimensionality to them, the Kernel PCA technique was used. The basic idea of this technique is to find a map function to a space of characteristics (generally of greater number of dimensions), which allows to simplify the classification. The disadvantage is that the problem gets complicated when increasing the number of dimensions in that the original data are represented. However, by means of the use of the functions Kernel we can obtain the advantages in the classification without the need of having to use or knowing the map function [14], this means, the work is directly done in the characteristics space by means of the map function as follows:

Applying the Kernel PCA technique the information is taken from the input space $x_{1}, \ldots, x_{n} \in \mathbb{R}^{m}$ to a space of characteristics $\mathrm{F}$ by means of a function of non-linear mapping and later the linear PCA is applied in F. The covariance matrix is calculated $C=1 / n \sum_{j=1}^{n} \Phi\left(x_{j}\right) \Phi\left(x_{j}\right)^{T}$, and the principal components are calculated solving the Eigenvalue problem, calculating the eigenvalues $\lambda \geq 0$ and the eigenvectors $V \backslash\{0\}$ and solving $\lambda V=C V=1 / n \sum_{j=1}^{n}\left(\Phi\left(x_{j}\right) V\right) \Phi\left(x_{j}\right)$.

Every solutions of $V$ with $\lambda \neq 0$ are inside of the mapped data set, for example, $V \in \operatorname{span}\left\{\Phi\left(x_{1}\right), \ldots, \Phi\left(x_{n}\right)\right\}$ this is expressed by means of $V=\sum_{i=1}^{n} \alpha_{i} \Phi\left(x_{i}\right)$. By multiplying both sides of $\lambda V=C V$ by $\Phi\left(x_{k}\right)$ (to $\left.k=1, \ldots, n\right)$ the eq. 5 is obtained.

$$
\lambda\left(\Phi\left(x_{k}\right) \cdot C V\right)=\left(\Phi\left(x_{k}\right) \cdot C V\right)
$$


Substituting the values of $C$ and $V$ in eq. 2 and considering a $n \times n$ matrix $K_{i j}=k\left(x_{i}, x_{j}\right)=\left(\Phi\left(x_{i}\right) \cdot \Phi\left(x_{j}\right)\right)$, the resulting equation can be reduced to $n \lambda K \alpha=K^{2} \alpha$, where $\alpha$ represents the column eigenvectors. In order to obtain the solutions of the equation 2 the eigenvalue problem $n \lambda \alpha=K \alpha$ is solved for nonnegative eigenvalues.

Once obtained the new space $\mathrm{F}$ it is necessary to centre the projected data (by means the kernel function $K$ ) using the following equation $\bar{K}=K-1_{n} K-K 1_{n}+1_{n} K 1_{n}$ [15], where $\left(1_{n}\right)_{i j}=1 / n$ that is a measure of proportion necessary for the normalization of $K$, and $n$ is the number of elements of the main $K$.

Now that we already have centred the data in $F$ we calculate the $\lambda$ 's eigenvalues of the matrix $K$, with their corresponding $\alpha$ 's eigenvectors. It is defined the optimal number of principal components to carry out the projection by means of this equation $\sum_{i=1}^{p} \lambda_{i} / \sum_{i=1}^{n} \lambda_{i}=60 \%$, which represents a variation percentage used in this work for the reconstruction of the original space, in this case $60 \%$, and $\mathrm{p}$ the number of necessary main components to obtain variation percentage.

To obtain a reduction of the images (matrix $K$ ), in terms of the edges (eigenvectors) that represent $60 \%$ of the space original, a projection function is applied multiplying the eigenvectors by the matrix $\mathrm{K} . Y_{\mathrm{i}}=\lambda_{1}^{\mathrm{T}}(\mathrm{K})$, where $i=1, \ldots, p$ are the vectors and $Y_{i}$ represent the images projected to the principal axes in the space of characteristic $\mathrm{F}$.

3. Captures of a new image: To test the classification a new image is captured in a different position to those of the obtained model, but in the same environment. With the purpose of being able to compare this image with those of the model the new image is projected to the eigenspace calculated by means of eq. 3 .

$$
(k P C)_{n}(x)=\left(V^{n} \cdot \Phi(x)\right)=\sum_{i=1}^{p} \alpha_{i}^{n} k\left(x_{i}, x\right)
$$

4. Bayesian Algorithm with an Active vision strategy for searching in F: The probability of generating a vector image $Y$ (random variable $Y$ ) in the eigenspace starting from a localization $T_{i}$ and an orientation $\Omega_{j}$ of the robot in a continuous environment (because the number of positions in the environment is infinite) is given for $P\left(Y \mid T_{i}, \Omega_{j}\right)$. By means of the rule of Bayes we can calculate the probability that the robot is in a position (localization and orientation) starting from an image $Y_{A}$ in the eigenspace:

$$
P\left(T_{i}, \Omega_{j} \mid Y_{A}\right)=P\left(Y_{A} \mid T_{i}, \Omega_{j}\right) P\left(T_{i}, \Omega_{j}\right) / \sum_{i j} P\left(Y_{A} \mid T_{i}, \Omega_{j}\right) P\left(T_{i}, \Omega_{j}\right)
$$

Where $P\left(T_{i}, \Omega_{j}\right)$ is the a priori probability of the location $T_{\mathrm{i}}$ and orientation $\Omega_{j}$.

Because the projected data to the eigenspace are samples taken from a population where it was considered the same mean (environment mean) and the same covariance matrix, by means of the central limit theorem is considered that their probability distribution approaches to a multivariate normal distribution. This allows us to calculate the probability $P\left(Y_{A} \mid T_{i}, \Omega_{j}\right)$ by means of the eq. 5 , considering as centre an image of the database (image projected to the eigenspace) $Y_{T_{i} \Omega_{i}}$. 


$$
P\left(Y_{A} \mid T_{i}, \Omega_{j}\right)=\exp \left[-1 / 2\left(Y_{A}-Y_{T_{i}, \Omega_{j}}\right)^{T} \Sigma^{-1}\left(Y_{A}-Y_{T_{i}, \Omega_{j}}\right)\right] / 2 \Pi^{k / 2}\left|\Sigma^{-1 / 2}\right|
$$

This formula allows us to know the distance between the vector $Y_{A}$ and a known vector in the database $Y_{T_{i}, \Omega_{j}}$. The probability that the robot is in a position $\mathrm{T}_{\mathrm{i}}$, starting from an image $Y$ observed from a localization $T_{i}$ and an orientation $\Omega_{j}$ can be calculated by means of $P\left(Y_{A} \mid T_{i}\right)=\sum_{i j} P\left(Y_{A} \mid T_{i}, \Omega_{j}\right) P\left(T_{i}, \Omega_{j}\right)$.

Applying the Bayes rule we can obtain the probability distribution of the independent location of the orientations $P\left(T_{i} \mid Y_{A}\right)=P\left(Y_{A} \mid T_{i}\right) P\left(T_{i}\right) / \sum_{i} P\left(Y_{A} \mid T_{i}\right) P\left(T_{i}\right)$.

If the probability of the observation overcomes a defined limit (50\% of probability) the robot is assigned to that position, otherwise an active vision strategy is used, active vision basically says that in accordance with the necessities are the actions taken, if the new image does not obtain a bigger probability than $50 \%$ (that which indicates that the current information is insufficient) a rotation movement of $\left(\mathrm{X}^{\circ}\right)$ is performed in order to obtain a new probability of a new image besides considering the current information:

$$
P\left(T_{i} \mid Y_{A}, Y_{B}\right)=P\left(Y_{A}, Y_{B} \mid T_{i}\right) P\left(T_{i}\right) / \sum_{i} P\left(Y_{A}, Y_{B} \mid T_{i}\right) P\left(T_{i}\right)
$$

Considering that $Y_{A}$ and $Y_{B}$ are conditionally independent events the equation 6 is rewritten as follow:

$$
P\left(T_{i} \mid Y_{A}, Y_{B}\right)=P\left(Y_{A} \mid T_{i}\right) P\left(Y_{B} \mid T_{i}\right) P\left(T_{i}\right) / \sum_{i} P\left(Y_{A} \mid T_{i}\right) P\left(Y_{B} \mid T_{i}\right) P\left(T_{i}\right)
$$

The success condition is that the obtained probability overcomes the defined limit, or the number of images settled down by location are captured until reaching the $360^{\circ}$, if these conditions are not completed it is determined that the current position of the robot is unknown.

\section{Experiments and Results}

\subsection{Algorithms for the Validation of the Proposed Method}

To measure the efficiency of the results obtained of the proposed techniques for the performance in the compression of the data we will use the results obtained by two techniques: Linear PCA which has been employed in the solution of the problem of the estimate of the position [16] considering a covariance matrix between the pixels, and APEX which although it has not been proven in this problem, possesses an important characteristic, according to its authors that it is the adaptation, that allows us to optimise the process of the extraction of the main components [17] [18], the results of the experiments are shown next.

Basically two types of experiments were carried out. The variations among the experiments consist in: the quantity of positions, initial orientation in the positions as well as the distance between each one of these. The quality of the solutions was measured according to the precision in the classification, that means, the number of test positions were classified in the positions of the environment model. 


\subsection{Experiment 1}

Conditions for the creation of the environment model. In order to not capture the information repeatedly, pixels variations from 50 to $70 \%$ were searched in the consecutive images, for this, 12 locations were defined with " 1 distance meter" among each one of them. In each one of the locations the robot rotates $360^{\circ}$ with the objective of capturing with the camera all the possible appearances of the environment from the current location. It was defined that the rotation intervals to capture an image are of $20^{\circ}$, this way, when we refer of orientations we refer to the $20^{\circ}$ that the robot should rotate before stopping, once detained a new image is captured, this image belongs to the location $T_{\mathrm{i}}$ and orientation $\Omega_{j}$ (see Fig. 4-a).

Table 1. Results of the realized tests in experiment 1. N.C. means that the test position was not classified in a position of the environment model.

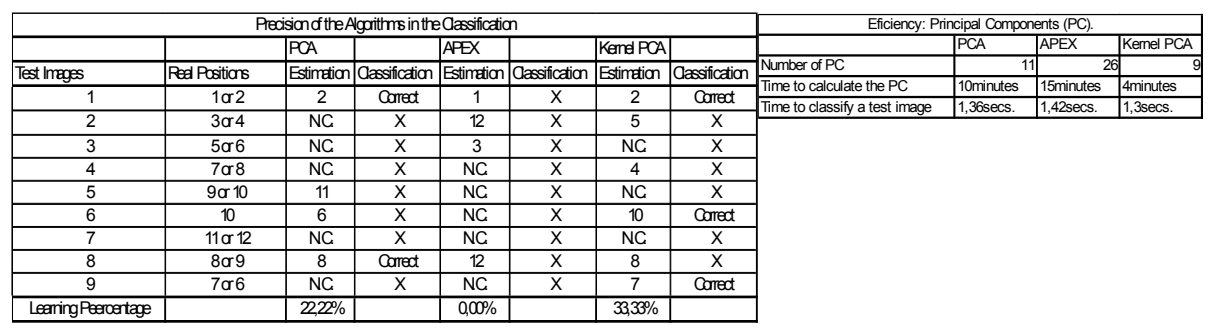

To prove the compression techniques as well as the classification algorithm the robot was positioned in 9 unknown random locations and initial orientation, but within the same environment, and the same process of compression of images was applied. The random positions allowed us to simulate the real conditions in which the position estimation would be done. The results in the experimentation are shown in the Table 1 .

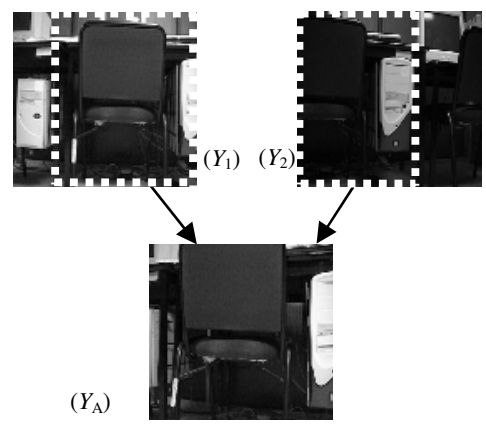

Fig. 2. Inconsistency in the classification. $Y_{1}$ Image belongs to the position 5 with $300^{\circ}$ orientation, $Y_{2}$ Image belongs to position 7 with $320^{\circ}$ of orientation. Test Image acquired between the position 5 and 7 with $310^{\circ}$ of orientation. 
In the percentages of correctly classified images the superiority of the Kernel PCA technique is appreciated with $33.33 \%$ in comparison with $0 \%$ (APEX) and $22.22 \%$ (PCA). Also the number of components required for the $60 \%$ reconstruction, and the time to calculate them was smaller using the Kernel PCA technique, because as is shown in the methodology, the matrix dimension employed to calculate PCA are of a smaller dimension ( $\mathrm{n} \times \mathrm{n}$ in the Kernel PCA (by means of a covariance among images) instead of $\mathrm{m} \times \mathrm{m}$ (covariance among pixels of the images) in the PCA and APEX, where $n$ is the quantity of images and $m$ is the pixel number in each image) using the technique Kernel PCA.

The reason for which the classification percentages were very low is: the variation that existed in the pixels of the images was such that in occasions only $30 \%$ of the image coincided with the next one, this produced ambiguity in calculating the ownership from an image to a well-known position. For this reason when varying the initial orientation in the test images the possibility that the test images did not coincide with some of the database increased; since the pixels of the test image could belong in a part to the beginning of an image and the rest to the final-center of another different image (see Fig. 2).

\subsection{Experiment 2}

The solution proposed for the problem in the experiment 1 is by means of the reduction of the pixels variation between an image and another, reducing the turn angle in each position (orientation angle in the positions), this way instead of having variations of between 50 and $70 \%$, variations of between 25 and $35 \%$ were obtained. To achieve this new positions ( 27 in total) and orientations ( 24 in total) were defined, the positions now would take half a meter with $15^{\circ}$ turns in each one of them, this allowed us to reduce the variations among the images. The distribution of the positions is shown in the Fig. 4-b. The results obtained in the experiment 2 are shown in the Table 2.

Table 2. Results of the realized tests in experiment 2. N.C. means that the test position was not classified in a position of the environment model.

\begin{tabular}{|c|c|c|c|c|c|c|c|c|c|c|c|}
\hline \multirow{2}{*}{\multicolumn{8}{|c|}{$\begin{array}{l}\text { Precision of the Algorithms in the Classification } \\
\text { PCA }\end{array}$}} & \multirow{2}{*}{\multicolumn{4}{|c|}{ Eficiency: Princial Components (PC). }} \\
\hline & & & & & & & & & & & \\
\hline Test Images & $\begin{array}{l}\text { Real Positions } \\
\end{array}$ & Estimation & Clasification & Estimation & \begin{tabular}{|l|l|} 
Clasification \\
\end{tabular} & Estimation & \begin{tabular}{|c|c|} 
Clasification \\
\end{tabular} & Number of PC & & 3 & \\
\hline 1 & 5 or 6 & 2 & Correct & 5 & Correct & 5 & Correct & Time to calculate the PC & 13 minutes & 19 minutes & 8.4 minutes \\
\hline 2 & 5 or 8 & N.C. & $x$ & 8 & Correct & 5 & Correct & Time to dassify a test image & 2,26 secs. & |2,43 secs. & 2,21 secs. \\
\hline 3 & 8 or 11 & 11 & Correct & 3 & $\bar{X}$ & 11 & Correct & & & & \\
\hline 4 & $\overline{12}$ & 12 & Correct & N.C. & $\bar{x}$ & $\overline{12}$ & Correct & & & & \\
\hline 5 & 14 or 17 & 11 & $\mathrm{x}$ & N.C. & Correct & N.C. & $x$ & & & & \\
\hline 6 & 17 or 18 or 19 & 10 & Correct & N.C. & $\bar{X}$ & 10 & Correct & & & & \\
\hline 7 & 20 or 23 & N.C. & $\mathrm{X}$ & N.C. & $\bar{X}$ & 23 & Correct & & & & \\
\hline 8 & 23 or 22 or 27 & 8 & Correct & 1 & $\bar{x}$ & 22 & Correct & & & & \\
\hline 9 & 25 or 26 & N.C. & $x$ & 12 & $\bar{x}$ & 19 & $\bar{X}$ & & & & \\
\hline 10 & 20 or 21 & 20 & Correct & 23 & $\bar{x}$ & 20 & Correct & & & & \\
\hline 11 & 17 or 16 & 2 & $\bar{x}$ & 16 & Correct & N.C. & $\bar{X}$ & & & & \\
\hline$\overline{12}$ & 14 or 15 & 11 & $\bar{x}$ & N.C. & $\bar{x}$ & 15 & Correct & & & & \\
\hline$\overline{13}$ & 10 or 11 & 15 & $x$ & 10 & Correct & 10 & Correct & & & & \\
\hline aning Perc & & $46,15 \%$ & & $38,47 \%$ & & $76,98 \%$ & & & & & \\
\hline
\end{tabular}

With a time average of classification of 2.64 seconds in each image, this time increased (according to the time classification average in the experiment 1) because the number of images was increased from 216 to 648. Although the quantity of images in the model increased, the precision of the three algorithms was also increased. 
To show the operation of the classification Bayesian algorithm in the Fig. 1 the behaviour of the probabilities of ownership of the position of test 8 is shown as mentioned in Table 2 in experiment 2 . In this position an image was captured with random initial orientation $\left(8^{\circ}\right)$, the initial orientation is different to the initial orientation of the positions in the model, the calculated probability did not overcome the established threshold $(50 \%)$, for this reason strategy of active vision was applied to register more information of the environment by means of capturing a new image in the same position but now in the orientation 1, rotating the robot $360 / 24^{\circ}$. Combining the information of the first one with the second image it is possible to overcome $50 \%$ of ownership in the position 22 although the biggest probability of ownership in the first image was obtained in the position 8 of the model (see Fig. 3).

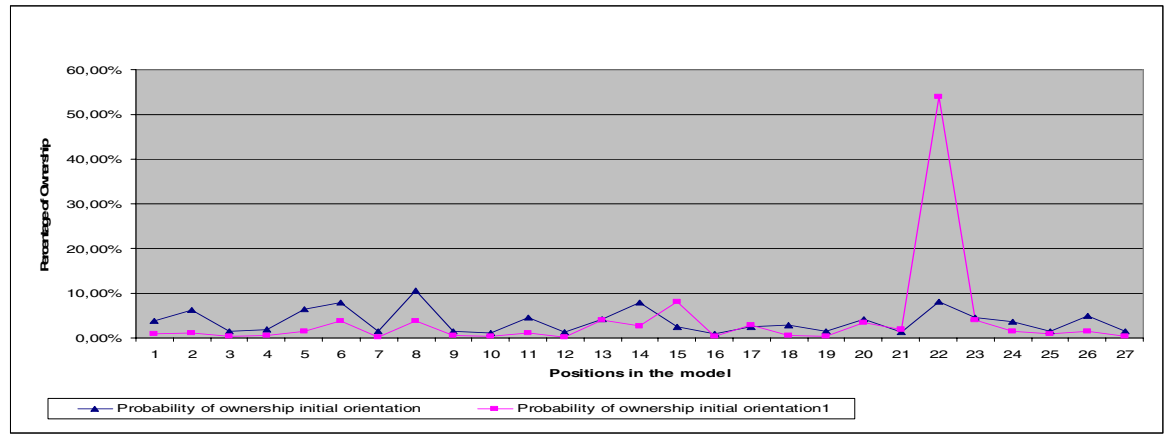

Fig. 3. Probabilities of Ownership for the classification of the position of test 8 . The graphic shows the behaviour of the Bayesian algorithm, in the first stage (initial orientation) the success condition wasn't reach; the active vision strategy was performed and a second image was taken. Considering all the available information (the two images), the ownership probability was computed. The result is the classification of the current position of the robot into the twentytwo position in the environment model.

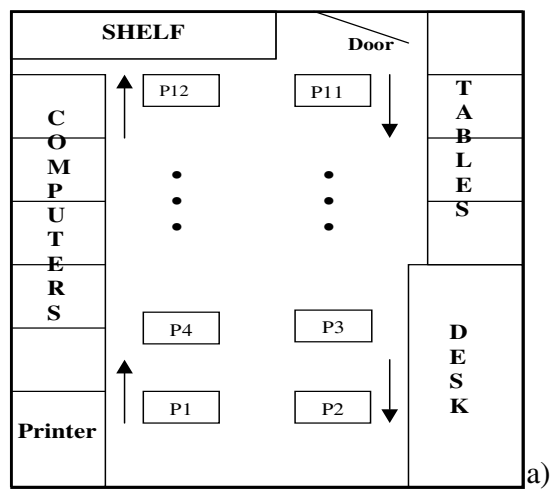

b)

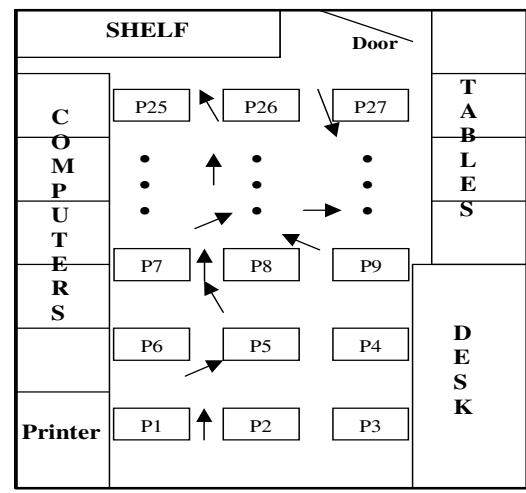

Fig. 4. Situation of the environment for the experiment 1 (a) and experiment 2 (b), the arrows indicate the orientation of the test positions. The numbers in the stalls indicate the position in the environment. The arrows indicate the initial orientation in each test position. 


\section{Conclusions}

The results obtained in the experimentation show that the Kernel PCA technique allowed us to improve the acting (bigger percentage of correct classifications, smaller time of calculation and number of required principal components) of the algorithms PCA and APEX. It can bee easily seen that the algorithm APEX requires more time to calculate the eigenvalues and the eigenvectors and the resulting components are not completely orthogonal, this explains the biggest quantity of necessary components to achieve the percentage.

\section{References}

1. Longuet-Higgins, H.C., Prazdny K.: The Interpretation of a Moving Retinal Image. In Proc. of the Royal Society B, London, 1980

2. Lourakis M.I.A.: Using Constraint Lines for Estimating Egomotion. Proc. of ACCV'2000, vol. 2, Taipei, Taiwan, Jan 2000, pp. 971 - 976.

3. Convertino G., Branca A., Distante A.: Focus of Expansion Estimation with a Neural Network. IEEE International Conference on Neural Networks, June 1996, pp. 1693 - 1697

4. Tomasi C., Kanade T.: Factoring images sequences into shape and motion. Proc. IEEE Workshop o Visual Motion, 1991, pp. 21 - 28.

5. Negadharipour S.: Direct Passive Navigation. PhD thesis, Department of Mechanical Engineering, MIT, 1986.

6. Daniilidis K., Spetsakis M.: Understanding noise sensitivity in structure from motion. Y. Aloimonos, editor, Visual Navigation, 1996, pp. $61-88$.

7. Burger W., Bhanu B.: Estimating 3D egomotion from perspective image sequence. Pattern Analysis and Machine Intelligence, IEEE, Honeywell Syst. \& Res. Center, Minneapolis, MN, nov 1990, pp. 1040-1058.

8. Murase H., Nayar S. K.: Visual learning and recognition of 3D objects from appearance. International Journal of Computer Vision, 14(1):5--24, Jan. 1995.

9. Cai Q., and Aggarwal J.K.: Tracking Human Motion in Structured Environments Using Distributed-Camera System. IEEE Transactions on Pattern Analysis, 1999, pp. 12411247.

10. Turk M., Pentland A.: Eigenfaces for recognition. J. Cog. Neurosci., 3(1), 1991, pp. 71-86.

11. Krose B. J., Bunschoten A. R.: Probabilistic Location by Appearance Models and Active Vision. Proceedings of the IEEE International Conference on Robotics and Automation,1999, pp. 2255-2260.

12. Yang M. H.: Kernel eigenfaces vs. kernel fisherfaces: Face recognition using kernel methods. in Proc. IEEE International Conference on Automatic Face and Gesture Recognition, pp. 215--220 (2002).

13. Pourraz F., Crowley J.: Continuity properties of the appearance manifold for mobile robot position estimation. In Proceedings of the 2nd IEEE Workshop on Perception for Mobile Agents, Ft. Collins, CO, IEEE Press, 2001, pp. 741-752.

14. Muller K., Mika S., Ratsch G., Tsuda K., Scholkopf B.: An introduction to kernel-based learning algorithms. IEEE Transactions on Neural Networks, March 2001, pp. 181-201.

15. Schölkopf B., Smola A. J., Müller K. R.: Nonlinear component analysis as a kernel eigenvalue problem. Neural Comput., vol. 10, 1998, pp.1299- 1319. 
16. Krose B. J. A., Bunschoten R.: Probabilistic Location by Appearance Models and Active Vision. Proceedings of the IEEE International Conference on Robotics and Automation, 1999, pp. 2255-2260.

17. Diamantaras K.I., Kung Y.: A Neural Network Learning Algorithm for Adaptive Principal Components Extraction. Prinsceton University, pp 860-864.

18. Oja E.: A simplified Neuron Model as a Principal Componet Analyser. J.Math. Biology, vol. 15, pp 267-273. 